\title{
Non-Small-Cell Lung Cancer: Treatment of Late Stage Disease: Chemotherapeutics and New Frontiers
}

\author{
Ronald J. Scheff, MD ${ }^{1}$ Bryan J. Schneider, MD ${ }^{1}$ \\ ${ }^{1}$ Division of Hematology/Oncology, Department of Internal Medicine, \\ Weill Cornell Medical College, New York, New York \\ Address for correspondence Bryan J. Schneider, MD, Division of \\ Hematology/Oncology, Department of Internal Medicine, Weill Cornell \\ Medical College, 525 East 68th Street, 3rd floor, Payson Pavilion, New \\ Semin Intervent Radiol 2013;30:191-198 \\ York, NY 10065 (e-mail: bjs2004@med.cornell.edu).
}

\begin{abstract}
Keywords

- non-small cell lung cancer

- chemotherapy

- systemic therapy

- targeted therapy

Systemic therapy should be considered in patients with advanced non-small cell lung cancer (NSCLC) who are no longer amenable to local therapies. Systemic therapy has been shown to improve survival and preserve quality of life in patients with a reasonable performance status. In unselected patients, the standard of care for initial therapy remains platinum-based chemotherapy. At progression, further treatment typically consists of the sequential administration of single-agent therapy, which has also been shown to improve survival and reduce cancer-related symptoms. Molecular biomarkers are essential to guide targeted agents. This analysis requires ample tumor DNA; thus adequate biopsy samples are critical to guide therapeutic options. More biomarkers are currently being validated and may potentially have specific targeted therapy. In the near future, it is likely that rapid multiplexed genotype testing will help reduce the need for large amounts of tumor for analysis and will promote personalized cancer therapy. We review recent changes in the definition of stage IV NSCLC and review current and future systemic therapeutic approaches for patients with advanced disease.
\end{abstract}

Objectives: Upon completion of this article, the reader will be able to list (1) the indications for treating non-small cell lung cancer with systemic therapy, (2) the most commonly used systemic therapies, (3) the need for adequate biopsy samples to ensure optimal tumor biomarker analysis, and (4) new targeted approaches based on tumor biomarkers.

Accreditation: This activity has been planned and implemented in accordance with the Essential Areas and policies of the Accreditation Council for Continuing Medical Education through the joint sponsorship of Tufts University School of Medicine (TUSM) and Thieme Medical Publishers, New York. TUSM is accredited by the ACCME to provide continuing medical education for physicians.

Credit: Tufts University School of Medicine designates this journal-based CME activity for a maximum of 1 AMA PRA Category 1 Credit $^{\mathrm{TM}}$. Physicians should claim only the credit commensurate with the extent of their participation in the activity.
Lung cancer is the leading cause of mortality, not just Advanced stage disease non-small cell lung cancer (NSCLC), the leading cause of cancer-related mortality in the United States and a leading cause of cancer-related mortality worldwide, remains a formidable clinical challenge. In the United States, $>220,000$ new cases of NSCLC are diagnosed annually. ${ }^{1}$ At the time of diagnosis, $\sim 40 \%$ of patients present with clinically evident metastatic disease that is beyond the curative potential of surgery or combined modality therapy. An additional $30 \%$ of patients present with locally advanced disease, most of whom will relapse with incurable disease despite aggressive combined modality approaches. Even among early stage patients who undergo surgery with curative intent, at least $30 \%$ will relapse, primarily at distant metastatic sites. These statistics indicate that upward of $80 \%$ of patients with NSCLC present with or eventually develop stage IV disease. Systemic therapy is the predominant treatment for patients with advanced NSCLC, based on
Issue Theme Pulmonary Malignancies; Guest Editors, Bradley B. Pua, MD and David C. Madoff, MD, FSIR
Copyright @ 2013 by Thieme Medical Publishers, Inc., 333 Seventh Avenue, New York, NY 10001, USA. Tel: +1(212) 584-4662.
DOI http://dx.doi.org/ 10.1055/s-0033-1342961. ISSN 0739-9529. 
clinical trials conducted over several decades that demonstrated improved survival and quality of life. ${ }^{2-4}$

\section{Stage IV Definition}

The staging system for NSCLC was most recently revised in 2009, the 7th edition of TNM staging. ${ }^{5}$ As with previous editions, the designation M1 indicates the presence of metastatic lung cancer in the contralateral lung or outside of the chest; however, M1 is now divided into M1a and M1b subcategories. M1a is defined as intrathoracic metastasis, where the spread of tumor is limited to the contralateral lung, pleura, and/or pericardium including malignant pericardial or pleural effusions. M1b indicates spread of tumor to at least one distant site, most typically bone, adrenal gland, liver, and/or brain. The designation of malignant pleural and/or pericardial effusion as M1a rather than T4 disease is a noteworthy change; such effusions are now indicative of stage IV rather than stage IIIB disease. Patients with intrathoracic metastases have a better survival rate compared with those with extrathoracic disease with a median of 10 versus 6 months, and 1-year survival of $45 \%$ versus $22 \%$, respectively $(p<0.0001) .{ }^{6}$ With rare exceptions, stage IV NSCLC is considered incurable, and the therapeutic approach is palliative rather than curative. ${ }^{7}$

\section{Assessment for Treatment}

NSCLC is no longer treated as a monolithic entity. It is increasingly imperative that specific tumor histology (squamous cell carcinoma versus adenocarcinoma versus large cell carcinoma) and predictive biomarkers, particularly the presence or absence of an epidermal growth factor receptor (EGFR) activating mutation or anaplastic lymphoma kinase (ALK) gene translocation, is established at the time of diagnosis. Such information facilitates the optimal selection of the most appropriate systemic therapy as discussed later in this article. When the decision has been made to proceed with systemic therapy, the choice of specific treatment is typically based on multiple factors including the patient's performance status, previous treatment, response to prior therapy, presence of predictive biomarkers, and underlying comorbid conditions.

\section{First-Line Systemic Therapy}

Multiple systemic therapeutic regimens are available for firstline use, ranging from traditional cytotoxic chemotherapy to new targeted agents. For patients with an adequate performance status, modern chemotherapy regimens can realistically achieve a tumor response rate of 20 to $30 \%$, median overall survival (OS) of 8 to 13 months, and a 1 -year survival rate of 30 to $50 \%$. A platinum doublet is the combination of either cisplatin or carboplatin with a third-generation cytotoxic (paclitaxel [Taxol], pemetrexed [Alimta], gemcitabine [Gemzar], docetaxel [Taxotere], or vinorelbine [Navelbine]) and remains the standard of care for initial systemic therapy. This two-drug combination is typically administered once or twice during a 3-week period, or cycle, for a total of four to six cycles.
Both cisplatin and carboplatin crosslink DNA through several mechanisms and interfere with mitosis. Data suggest that cisplatin may have a modest efficacy advantage over carboplatin in the treatment of NSCLC. ${ }^{8}$ However, the toxicity of cisplatin may be intolerable for many patients, particularly in the palliative setting, where quality of life is paramount. Compared with carboplatin, cisplatin is associated with a greater risk and degree of nausea, alopecia, renal toxicity, ototoxicity, and peripheral neuropathy. Carboplatin is more myelosuppressive, but given the superior tolerability, it has now become the more commonly used platinum compound for the treatment of advanced NSCLC in the United States.

Selection of the specific platinum doublet remains discretionary because the data would suggest similar clinical benefit among the regimens but different toxicity profiles. The Eastern Cooperative Oncology Group (ECOG) 1594 study confirmed equivalent efficacy among four different platinum doublets. ${ }^{9}$ In this seminal clinical trial, 1207 patients with advanced NSCLC were prospectively randomized to receive one of four platinum doublet chemotherapy regimens: cisplatin plus paclitaxel or docetaxel (taxanes that induce a mitotic block by stabilizing microtubules); cisplatin plus gemcitabine (a nucleotide analog that inhibits essential enzymes for DNA synthesis and function); or carboplatin plus paclitaxel. Patients in all four treatment arms continued therapy until disease progression or intolerable toxicity. Median OS for all enrolled patients was 8.0 months and did not differ statistically among the treatment arms. Similarly, 1 - and 2-year survival rates were $34 \%$ and $12 \%$, respectively, and did not vary significantly by treatment arm. Patients treated on the carboplatin plus paclitaxel arm experienced less toxicity compared with the patients who received cisplatin-based therapy. Patients with a poor performance status tolerated treatment poorly and had a lower median OS (3.9 months) when compared with patients with a good performance status ( 7.1 to 10.8 months) regardless of the treatment arm, underscoring the importance of assessing performance status prior to initiation of therapy.

Non-platinum-containing regimens such as gemcitabine plus a taxane or vinorelbine (a vinca alkaloid that inhibits mitosis) have also been studied as first-line treatment of stage IV NSCLC. ${ }^{10,11}$ However, there was no major advantage in the adverse event profile of non-platinum regimens for them to be favored over the use of platinum-based combinations. For selected patients who are unlikely to tolerate platinum-based therapy, the non-platinum regimens are acceptable therapeutic alternatives.

Tumor histology has become an important factor when selecting first-line therapy. In a large phase 3 clinical trial, 1725 patients with advanced NSCLC were randomized to cisplatin plus pemetrexed (an antifolate antimetabolite that inhibits multiple enzymes involved in purine and pyrimidine synthesis) versus cisplatin plus gemcitabine as initial systemic therapy. ${ }^{12,13}$ Although the median OS in both treatment arms was 10.3 months, a preplanned assessment of survival between the different histologic subtypes revealed clinically relevant differences in efficacy between the two treatment arms. Patients with adenocarcinoma demonstrated improved 
median OS with pemetrexed compared with gemcitabine (12.6 versus 10.9 months). Conversely, patients with squamous cell histology had improved median survival with gemcitabine over pemetrexed (10.8 versus 9.4 months). This trial and other studies identified that squamous cell carcinoma of the lung did not respond to pemetrexed compared with other chemotherapy; pemetrexed was therefore restricted to patients with "non-squamous-cell" NSCLC.

\section{Maintenance Therapy}

The optimal duration of first-line therapy remains a subject of clinical controversy. The standard of care for initial treatment of advanced NSCLC has been four to six cycles of platinumbased chemotherapy followed by close observation. This approach was based on studies that suggested increased toxicity with no improved clinical benefit when the platinum doublet was continued until disease progression. ${ }^{14,15}$ Unfortunately, most patients will progress within 6 months of completing initial chemotherapy. Recently, two maintenance treatment strategies have emerged: continuation maintenance (an agent used during front-line therapy is continued until disease progression) and switch maintenance (a different agent is initiated upon completion of four cycles of platinum-based chemotherapy).

In the ECOG 4599 trial, 878 patients with advanced NSCLC were randomized to receive up to six cycles of carboplatin plus paclitaxel with or without the addition of bevacizumab (Avastin), a monoclonal antibody that targets vascular endothelial growth factor and is thought to impair tumor angiogenesis. $^{16}$ Patients with squamous histology, brain metastasis, hemoptysis, or other indications of excess bleeding risk were excluded. Patients randomized to the bevacizumab arm received it concurrently with chemotherapy for six cycles and then continued bevacizumab monotherapy until disease progression. The addition of bevacizumab to chemotherapy followed by maintenance bevacizumab significantly improved response rate (35\% versus 15\%; $p<0.001$ ), median progression-free survival (PFS) (6.2 versus 4.5 months), and median OS (12.3 versus 10.3 months; hazard ratio [HR]: $0.79, p=0.03$ ) compared with chemotherapy alone. Toxicity was manageable with increased neutropenia and bleeding risk with the addition of bevacizumab. Subsequently, it was shown that bevacizumab may be safely administered to patients with brain metastases who have been treated with surgical resection or radiation therapy. ${ }^{17}$

In the FLEX trial, 1125 patients with advanced NSCLC with tumors that expressed the EGFR protein were randomized to receive up to six cycles of cisplatin plus vinorelbine with or without cetuximab (Erbitux), a monoclonal antibody that targets EGFR. ${ }^{18}$ Patients randomized to receive cetuximab started it concurrently with chemotherapy and continued it beyond the six chemotherapy cycles until disease progression or unacceptable toxicity. Patients randomized to cetuximab experienced a modest improvement in median OS compared with patients who received chemotherapy alone (11.3 versus 10.1 months). Unfortunately, a similar phase 3 randomized trial of carboplatin and paclitaxel with or without cetuximab in advanced NSCLC did not demonstrate a survival benefit, and therefore cetuximab has not been routinely used in clinical practice. ${ }^{19}$

Continuation maintenance chemotherapy has also been shown to proffer some benefit. In the recently reported PARAMOUNT trial, 539 patients with advanced nonsquamous NSCLC whose disease did not progress following four cycles of cisplatin plus pemetrexed were randomized to either continuation maintenance therapy of single-agent pemetrexed or placebo. ${ }^{20}$ A statistically significant improvement in median OS (16.9 versus 14.0 months) was demonstrated in the arm receiving pemetrexed. Although the survival appears to be much longer compared with the previous studies mentioned, it should be noted that patients were only eligible if they did not demonstrate disease progression during the initial four cycles of cisplatin/pemetrexed chemotherapy, thus excluding patients with more aggressive disease.

Other studies have evaluated a switch maintenance strategy. The JMEN trial enrolled 663 patients with advanced NSCLC whose disease had not progressed after four cycles of a non-pemetrexed-containing platinum doublet, and they were randomized to receive immediate pemetrexed therapy (given until progression) or placebo. ${ }^{21}$ Treatment with maintenance pemetrexed resulted in statistically significant improvements in both median PFS (4.3 versus 2.6 months) and OS (13.4 versus 10.6 months) compared with patients receiving placebo, with manageable toxicity. Among the 481 patients with nonsquamous histology, median OS from the time of randomization was 15.5 months with maintenance pemetrexed versus 10.3 months for patients who received placebo.

Fidias et al conducted a study in patients with advanced NSCLC who did not progress after four cycles of carboplatin plus gemcitabine and were randomized to either immediate therapy with docetaxel or docetaxel at the time of disease progression. ${ }^{22}$ A statistically significant improvement in median PFS was identified for patients receiving immediate docetaxel (5.7 versus 2.7 months; $p=0.0001$ ), with a trend toward improved median OS (12.3 versus $9.7 ; p=0.0853$ ).

The use of targeted agents may also be an effective switch maintenance strategy. In the SATURN trial, 889 patients with advanced NSCLC who did not progress after four cycles of platinum-based chemotherapy were randomized to receive placebo or immediate erlotinib (Tarceva, an orally administered EGFR tyrosine kinase inhibitor (TKI) that blocks tumor signal transduction). ${ }^{23,24}$ From the time of randomization, a modest improvement in both median PFS (2.8 versus 2.6 months) and median OS (12 versus 11 months) were demonstrated in the group treated with erlotinib. Rash and diarrhea were the main toxicities but were manageable and rarely led to discontinuation of treatment.

Despite these compelling data that suggest a real survival benefit with maintenance therapy, these trials have been heavily criticized for their design. The bevacizumab trial did not include a group that received chemotherapy with bevacizumab for six cycles followed by observation; thus the benefit of maintenance bevacizumab was never proven. In the switch maintenance trials, only $20 \%$ of patients in the control arms ever received the study agent (pemetrexed or erlotinib) as subsequent therapy, leaving skeptics to wonder what the 
overall survival benefit would have been if these agents were offered sometime during the course of treatment. Indeed, in the immediate versus delayed docetaxel trial, the median OS was identical as long as the patient received docetaxel ( $40 \%$ in the delayed arm never received the docetaxel due to symptomatic deterioration). It did not seem to matter clinically if the treatment was given immediately following the induction therapy or at progression as long as the patient received the therapy at some point.

\section{Treatment of Relapsed Disease}

The treatment of advanced NSCLC after the failure of initial therapy remains a challenge. The major goal in this setting needs to be preservation of quality of life. Several large phase 3 trials have demonstrated both improvement in survival and quality of life with the use of single-agent therapy in the setting of relapse. ${ }^{25-28}$ Three agents are currently approved for use in the second line setting by the U.S. Food and Drug Administration: docetaxel, pemetrexed, and erlotinib. Other chemotherapeutic agents such as gemcitabine and vinorelbine have been extensively investigated in the relapsed/ refractory setting and are commonly used in clinical practice. $^{29-31}$ Although combination chemotherapy with two or more agents may improve the tumor response rate, this approach has not been shown to improve survival or enhance quality of life in patients who progressed during or after front-line platinum-based chemotherapy, and the additive toxicity limits its use outside of a clinical trial. ${ }^{32}$

Docetaxel was the first chemotherapy approved for second-line therapy; side effects of this agent when given as monotherapy typically include cytopenias, fatigue, fluid retention, and nail changes. Two randomized controlled phase 3 trials have evaluated the efficacy of docetaxel in patients with advanced NSCLC who had progressed after prior chemotherapy. ${ }^{25,33}$ In a study reported by Shepherd et al, ${ }^{25} 204$ patients who had been previously treated with a platinum-based regimen were randomized to receive docetaxel or best supportive care. Patients who received docetaxel had a response rate of $5.5 \%$ and demonstrated a significant improvement in both median survival (7.5 versus 4.6 months) and 1-year survival (37\% versus $19 \%$ ) compared with those assigned to best supportive care. Two separate reports evaluated the quality of life of patients on these randomized trials. ${ }^{34,35}$ Both reports demonstrated a statistically significant trend favoring the docetaxel arm, with patients reporting less pain, fatigue, and better overall quality of life when compared with patients on the control arm.

A phase 3 trial comparing pemetrexed versus docetaxel in patients with relapsed or refractory NSCLC included 571 patients who had progressed after one prior regimen for advanced NSCLC. Response rates (9.1\% versus $8.8 \%$ ) and median OS (8.3 versus 7.9 months) were similar in the pemetrexed and docetaxel arms, respectively. However, toxicity favored the pemetrexed arm, with significantly less neutropenia (5\% versus $40 \%$ ), neutropenic fever ( $2 \%$ versus $13 \%$ ), and peripheral neuropathy (3\% versus $8 \%$ ), making it a preferred agent in the relapsed setting if not used as initial therapy.
Erlotinib does not induce myelosuppression, making it an attractive option for patients who have been heavily treated with myelosuppressive chemotherapy. The efficacy of erlotinib monotherapy was evaluated in a phase 3 trial that enrolled 731 patients with progressive NSCLC after initial platinum-based therapy, and they were randomized to either erlotinib or best supportive care alone. The response rate was $8.9 \%$, and the median OS was 6.7 months with erlotinib versus 4.7 months with best supportive care. Gefitinib (Iressa) is another EGFR-TKI that is not approved in the United States presently but is commonly used in Europe and Asia. The combination of erlotinib or gefitinib with chemotherapy has been investigated in four large randomized prospective phase 3 trials, and unfortunately no improvement in survival was identified with addition of the targeted agent to standard cytotoxic therapy. ${ }^{36-39}$

\section{Personalized Therapy}

We have entered the era of targeted therapy in oncology with the identification of cancer genetic "driver mutations" that define unique molecular subsets of NSCLC. These genetic derangements allow treating physicians to tailor therapy based on these "actionable mutations." The first actionable mutation in NSCLC was the identification of EGFR somatic mutations: deletions in exon 19 and the L858R point mutation. These EGFR mutations are more commonly seen in female Asian patients with adenocarcinoma and little or no smoking history. In an unselected population of NSCLC patients in the United States, $10 \%$ will have tumors that harbor an EGFRactivating mutation. These tumors are exquisitely sensitive to erlotinib, with response rates that exceed $80 \%$ that may afford a PFS beyond 1 year. ${ }^{40}$ The pivotal IPASS study evaluated never or light smokers (less than 10 pack-years and quit $>15$ years ago) with advanced NSCLC. This was an enriched patient population for EGFR mutant-positive disease and indeed, $60 \%$ of tumors analyzed had an EGFR-activating mutation. Patients were randomized to initial chemotherapy or to gefitinib, and those with an EGFR mutation who received gefitinib demonstrated significant improvement of PFS compared with initial chemotherapy (HR for progression or death: 0.48 ; 95\% confidence interval $[\mathrm{CI}], 0.36$ to $0.64 ; p<0.001$ ). Interestingly, the patients without an EGFR-activating mutation demonstrated a shorter PFS with gefitinib compared with chemotherapy (HR for progression or death: $2.85 ; 95 \% \mathrm{CI}, 2.05$ to $3.98 ; p<0.001$ ), suggesting that EGFR mutation-negative patients should not receive an EGFR-TKI as initial therapy. Not surprisingly, the median OS was similar between the two arms ( $\sim 18$ months) due to crossover effect (i.e., patients initially treated with chemotherapy received the gefitinib as second-line therapy and vice versa).

Five randomized phase 3 studies have evaluated first-line treatment with an EGFR-TKI versus standard chemotherapy in patients with EGFR mutation-positive NSCLC. ${ }^{41-45}$ The initial use of an EGFR-TKI improved PFS from 4.5 to 7 months to 9 to 13 months compared with platinum-based chemotherapy. Median OS was similar between the treatment arms again due to crossover effect and was in the 20 - to 30 -month range. It 
is rapidly becoming standard practice to test advanced NSCLC for an EGFR-activating mutation at the initial diagnosis. Presently, reverse transcriptase-polymerase chain reaction (RT-PCR) is required to identify EGFR mutations, an analysis that requires ample tumor DNA for an accurate assessment.

The next actionable genetic abnormality validated in NSCLC was the anaplastic lymphoma kinase (ALK) fusion oncogene. This has been identified by fluorescent in situ hybridization (FISH) analysis in $\sim 3$ to $5 \%$ of patients with NSCLC and is also typically found in never or light smoking patients with adenocarcinoma. Crizotinib (Xalkori) is an inhibitor of the ALK tyrosine kinase and was approved in late 2011 for the treatment of ALK-positive NSCLC based on two early-phase studies in heavily pretreated patients with ALK-positive tumors. ${ }^{46,47}$ Over 250 ALK-positive patients were enrolled, many of whom had received more than four previous lines of therapy, and it demonstrated an impressive response rate of 53 to $61 \%$ and median PFS of 8.5 to 9.7 months. Side effects typically included vision changes, nausea, vomiting, and peripheral edema, but were they manageable and rarely resulted in the discontinuation of treatment. Most importantly, a clinically meaningful improvement in quality of life was identified with improvement in pain, dyspnea, cough, and fatigue from baseline. Further phase 3 studies in both the first-line and second-line setting are underway to validate these findings.

KRAS mutant lung cancer may confer a poorer prognosis compared with other NSCLCs, and data suggest these tumors demonstrate increased resistance to both platinum-based chemotherapy and EGFR-TKIs. ${ }^{48,49}$ Approximately 15 to $30 \%$ of lung adenocarcinomas harbor a KRAS mutation, but unlike EGFR and ALK, KRAS mutations have been difficult to target therapeutically. Recently, the approach of targeting MEK (which is a downstream effector protein in the cell signaling pathway) has shown promise as a therapeutic option. In a randomized phase 2 study, 87 relapsed patients with KRAS mutation-positive NSCLC were treated with standard docetaxel or docetaxel plus the MEK inhibitor selumetinib. ${ }^{50}$ No responses were seen with docetaxel alone versus a $37 \%$ response rate with the addition of selumetinib. A statistically significant improvement in median PFS was identified (2.1 months versus 5.3 months; $p=0.014$ ) in addition to a trend toward improvement in median OS (5.2 months versus 9.4 months; $p=0.21$ ).

Several other NSCLC driver mutations/gene translocations are currently under investigation including ROS1/RET rearrangements and BRAF mutations, all of which will have a specific targeted therapy. For example, tumors that harbor a ROS1 fusion oncogene appear to also be sensitive to crizotinib. ${ }^{51,52}$ In one study, 14 patients with ROS1-positive tumors were treated with crizotinib and demonstrated a response rate of $54 \%$ and median duration of response of 13 weeks. A novel KIF5B-RET fusion oncogene has recently been identified, and a phase 2 study with the RET inhibitor XL 184 (Exelixis) in patients with tumors that harbor RET fusion oncogenes is in development. ${ }^{53}$ BRAF mutations, commonly identified in malignant melanoma, are found in 3\% of NSCLC, and case reports would suggest these patients may respond to the BRAF inhibitor vemurafenib. ${ }^{54,55}$ In the near future, the validation of many more biomarkers to guide therapy may be seen including HER2 mutations; MET overexpression; FDFR1 amplification; DDR2 mutation; MEK1 mutation; and AKT1, PTEN, and PIK3CA alterations.

Finally, preliminary data suggest that NSCLC may respond to novel immunotherapy approaches. PD-1 is an immune checkpoint receptor commonly found on $\mathrm{T}$ cells. This cell receptor is involved in $\mathrm{T}$-cell regulation and is primarily expressed by activated memory and regulatory $\mathrm{T}$ cells. $\mathrm{T}$ cells are downregulated when PD-1 binds to PD-L1/L2 expressed by dendritic, parenchymal, and tumor cells. Tumor cells have been shown to evade the immune system by expressing PD-L1, and expression of this receptor can be identified by immunohistochemistry. A recent phase 1 study evaluating the PD-1 inhibitor BMS-936558 included 75 patients with advanced NSCLC. ${ }^{56}$ The objective response rate was $18 \%$ with a response duration that ranged from 2 to $\geq 30$ months. Importantly, responses were seen in both squamous and nonsquamous cell histology. The most common severe side effects included fatigue, pneumonitis, and elevated hepatic transaminases. Given these intriguing results, it is likely that anti-PD1 will become a validated therapeutic approach for patients with NSCLC.

\section{Challenges}

In routine clinical practice, the process of testing for tumor driver mutations remains a challenge on many levels. Frequently, an initial biopsy of NSCLC will not have enough tumor cells for analysis given the amount of tumor DNA required for RT-PCR and FISH analysis to identify EGFR mutations and ALK translocations, respectively. A repeat biopsy is often required to obtain adequate tissue that may lead to increased risk and cost for the patient. Although a fineneedle aspiration with grossly visible material may yield enough tumor cells for analysis, two to five core biopsies are preferable whenever possible.

The specific patient population to analyze is controversial at the present time. Some centers advocate for the testing of all patients with NSCLC; others suggest that this expensive and labor-intensive analysis should be restricted to never or light smoking patients with adenocarcinoma who are most likely to have NSCLC that harbors an actionable driver mutation. The National Comprehensive Cancer Network guidelines recommend the evaluation for EGFR-activating mutations and ALK gene translocations in all patients with nonsquamous NSCLC given the extreme rarity of EGFR/ALK positivity in squamous cell carcinoma. ${ }^{57}$ In the future, next-generation sequencing technologies should enhance the analysis of cancer genomes and streamline this process. ${ }^{58} \mathrm{High}$-throughput sequencing technologies will allow the rapid identification of actionable mutations and guide the use of an everincreasing number of targeted agents.

Despite the identification of these activating mutations, patients will ultimately develop resistance to therapy due to a variety of molecular mechanisms that may include increased gene copy number of the mutated driver gene, a secondary 
resistance mutation, and/or activation of bypass signaling pathways. Currently, trials are investigating these mechanisms with the goal to develop agents that will overcome resistance. Repeat biopsies at the time of progression may be needed to identify the specific mechanism of resistance and guide further treatment options. Finally, treatment of squamous cell carcinoma and small cell lung cancer has not had any major advances in the past several years, although efforts are underway to identify potentially targetable driver mutations in patients with these difficult-to-treat histologies. ${ }^{59}$

\section{Conclusion}

Chemotherapy for advanced NSCLC has been shown consistently to improve survival and promote quality of life in carefully selected patients. Empirical treatment approaches are no longer optimal, and many centers have embraced validated biomarkers to guide both the use of chemotherapy and newer targeted agents. It is hoped that the continued identification of targetable driver mutations and other biomarkers will have a favorable impact on the prognosis and quality of life of all patients with cancer, including those with advanced NSCLC. In the near future, multiplexed genotype testing will allow large panels of relevant genotypes to be analyzed from a single tumor sample. ${ }^{60}$ This will bring us closer to the so-called holy grail of oncology: truly personalized cancer therapy. Unfortunately, despite recent advances, advanced NSCLC remains a terminal disease. Thus optimal supportive care, with or without further anticancer therapy, needs to be the primary goal of managing physicians. The treating team should always consider enrollment on clinical trials, including phase 1 trials, for patients with good performance status and advanced NSCLC.

\section{References}

1 American Cancer Society. Cancer facts and figures 2012. Atlanta, GA: 2012. Available at: http://www.cancer.org/acs/groups/content/@epidemiologysurveilance/documents/document/acspc031941.pdf

2 Rapp E, Pater JL, Willan A, et al. Chemotherapy can prolong survival in patients with advanced non-small-cell lung cancer-report of a Canadian multicenter randomized trial. J Clin Oncol 1988;6 (4):633-641

3 Marino P, Pampallona S, Preatoni A, Cantoni A, Invernizzi F. Chemotherapy vs supportive care in advanced non-small-cell lung cancer. Results of a meta-analysis of the literature. Chest 1994;106(3):861-865

4 Non-Small Cell Lung Cancer Collaborative Group. Chemotherapy in non-small cell lung cancer: a meta-analysis using updated data on individual patients from 52 randomised clinical trials. BMJ 1995;311(7010):899-909

5 Rami-Porta R, Crowley JJ, Goldstraw P. The revised TNM staging system for lung cancer. Ann Thorac Cardiovasc Surg 2009;15(1):4-9

6 Postmus PE, Brambilla E, Chansky K, et al; International Association for the Study of Lung Cancer International Staging Committee; Cancer Research and Biostatistics; Observers to the Committee; Participating Institutions. The IASLC Lung Cancer Staging Project: proposals for revision of the $\mathrm{M}$ descriptors in the forthcoming (seventh) edition of the TNM classification of lung cancer. J Thorac Oncol 2007;2(8):686-693
7 Tanvetyanon T, Robinson LA, Schell MJ, et al. Outcomes of adrenalectomy for isolated synchronous versus metachronous adrenal metastases in non-small-cell lung cancer: a systematic review and pooled analysis. J Clin Oncol 2008;26(7):1142-1147

8 Ardizzoni A, Boni L, Tiseo M, et al; CISCA (CISplatin versus CArboplatin) Meta-analysis Group. Cisplatin- versus carboplatinbased chemotherapy in first-line treatment of advanced nonsmall-cell lung cancer: an individual patient data meta-analysis. J Natl Cancer Inst 2007;99(11):847-857

9 Schiller JH, Harrington D, Belani CP, et al; Eastern Cooperative Oncology Group. Comparison of four chemotherapy regimens for advanced non-small-cell lung cancer. N Engl J Med 2002;346 (2):92-98

10 Herbst RS, Khuri FR, Lu C, et al. The novel and effective nonplatinum, nontaxane combination of gemcitabine and vinorelbine in advanced nonsmall cell lung carcinoma: potential for decreased toxicity and combination with biological therapy. Cancer 2002;95 (2):340-353

11 Jiang J, Liang X, Zhou X, Huang R, Chu Z, Zhan Q. Non-platinum doublets were as effective as platinum-based doublets for chemotherapy-naïve advanced non-small-cell lung cancer in the era of third-generation agents. J Cancer Res Clin Oncol 2013;139(1): 25-38

12 Molina JR, Adjei AA. The role of Pemetrexed (Alimta, LY231514) in lung cancer therapy. Clin Lung Cancer 2003;5(1):21-27

13 Scagliotti GV, Parikh P, von Pawel J, et al. Phase III study comparing cisplatin plus gemcitabine with cisplatin plus pemetrexed in chemotherapy-naive patients with advanced-stage non-smallcell lung cancer. J Clin Oncol 2008;26(21):3543-3551

14 Socinski MA, Schell MJ, Peterman A, et al. Phase III trial comparing a defined duration of therapy versus continuous therapy followed by second-line therapy in advanced-stage IIIB/IV non-small-cell lung cancer. J Clin Oncol 2002;20(5):1335-1343

15 Soon YY, Stockler MR, Askie LM, Boyer MJ. Duration of chemotherapy for advanced non-small-cell lung cancer: a systematic review and meta-analysis of randomized trials. J Clin Oncol 2009;27 (20):3277-3283

16 Sandler A, Gray R, Perry MC, et al. Paclitaxel-carboplatin alone or with bevacizumab for non-small-cell lung cancer. N Engl J Med 2006;355(24):2542-2550

17 Socinski MA, Langer CJ, Huang JE, et al. Safety of bevacizumab in patients with non-small-cell lung cancer and brain metastases. J Clin Oncol 2009;27(31):5255-5261

18 Pirker R, Pereira JR, Szczesna A, et al; FLEX Study Team. Cetuximab plus chemotherapy in patients with advanced non-small-cell lung cancer (FLEX): an open-label randomised phase III trial. Lancet 2009;373(9674):1525-1531

19 Lynch TJ, Patel T, Dreisbach L, et al. Cetuximab and first-line taxane/carboplatin chemotherapy in advanced non-small-cell lung cancer: results of the randomized multicenter phase III trial BMS099. J Clin Oncol 2010;28(6):911-917

20 Paz-Ares L, De Marinis F, Dediu M, et al. PARAMOUNT: Final overall survival (OS) results of the phase III study of maintenance pemetrexed (pem) plus best supportive care (BSC) versus placebo (plb) plus BSC immediately following induction treatment with pem plus cisplatin (cis) for advanced nonsquamous (NS) non-small cell lung cancer (NSCLS). J Clin Oncol 2012;30(suppl):Abstract LBA7507

21 Ciuleanu T, Brodowicz T, Zielinski C, et al. Maintenance pemetrexed plus best supportive care versus placebo plus best supportive care for non-small-cell lung cancer: a randomised, doubleblind, phase 3 study. Lancet 2009;374(9699):1432-1440

22 Fidias PM, Dakhil SR, Lyss AP, et al. Phase III study of immediate compared with delayed docetaxel after front-line therapy with gemcitabine plus carboplatin in advanced non-small-cell lung cancer. J Clin Oncol 2009;27(4):591-598

23 Sridhar SS, Seymour L, Shepherd FA. Inhibitors of epidermalgrowth-factor receptors: a review of clinical research with a focus on non-small-cell lung cancer. Lancet Oncol 2003;4(7):397-406 
24 Cappuzzo F, Ciuleanu T, Stelmakh L, et al; SATURN investigators. Erlotinib as maintenance treatment in advanced non-small-cell lung cancer: a multicentre, randomised, placebo-controlled phase 3 study. Lancet Oncol 2010;11(6):521-529

25 Shepherd FA, Dancey J, Ramlau R, et al. Prospective randomized trial of docetaxel versus best supportive care in patients with nonsmall-cell lung cancer previously treated with platinum-based chemotherapy. J Clin Oncol 2000;18(10):2095-2103

26 Hanna N, Shepherd FA, Fossella FV, et al. Randomized phase III trial of pemetrexed versus docetaxel in patients with non-small-cell lung cancer previously treated with chemotherapy. J Clin Oncol 2004;22(9):1589-1597

27 Shepherd FA, Rodrigues Pereira J, Ciuleanu T, et al; National Cancer Institute of Canada Clinical Trials Group. Erlotinib in previously treated non-small-cell lung cancer. N Engl J Med 2005;353(2): 123-132

28 Noble J, Ellis PM, Mackay JA, Evans WK; Lung Cancer Disease Site Group of Cancer Care Ontario's Program in Evidence-based Care. Second-line or subsequent systemic therapy for recurrent or progressive non-small cell lung cancer: a systematic review and practice guideline. J Thorac Oncol 2006;1(9):10421058

29 Crinò L, Mosconi AM, Scagliotti G, et al. Gemcitabine as second-line treatment for advanced non-small-cell lung cancer: a phase II trial. J Clin Oncol 1999;17(7):2081-2085

30 Lara PN Jr, Gumerlock PH, Mack PC, et al. Gemcitabine in patients with non-small-cell lung cancer previously treated with platinumbased chemotherapy: a phase II California cancer consortium trial. Clin Lung Cancer 2004;6(2):102-107

31 Santoro A, Maiorino L, Santoro M. Second-line with vinorelbine in the weekly monochemotherapy for the treatment of advanced non-small cell lung cancer. Lung Cancer 1994;11:S130

32 Di Maio M, Chiodini P, Georgoulias V, et al. Meta-analysis of singleagent chemotherapy compared with combination chemotherapy as second-line treatment of advanced non-small-cell lung cancer.J Clin Oncol 2009;27(11):1836-1843

33 Fossella FV, DeVore R, Kerr RN, et al. Randomized phase III trial of docetaxel versus vinorelbine or ifosfamide in patients with advanced non-small-cell lung cancer previously treated with platinum-containing chemotherapy regimens. The TAX 320 Non-Small Cell Lung Cancer Study Group. J Clin Oncol 2000;18(12):23542362

34 Miller VA, Fossella FV, DeVore R, et al. Docetaxel benefits lung cancer symptoms and quality of life in a randomized phase III study of non-small cell lung cancer patients previously treated with platinum-based therapy. Proc Am Soc Clin Oncol 1999; 18:491a

35 Dancey J, Shepherd F, Ramlau R, et al. Quality of life assessment in a randomized study of taxotere versus best supportive care in nonsmall cell lung cancer patients previously treated with platinumbased chemotherapy. Proc Am Soc Clin Oncol 1999;18:491a

36 Giaccone G, Herbst RS, Manegold C, et al. Gefitinib in combination with gemcitabine and cisplatin in advanced non-small-cell lung cancer: a phase III trial-INTACT 1. J Clin Oncol 2004;22(5):777784

37 Herbst RS, Giaccone G, Schiller JH, et al. Gefitinib in combination with paclitaxel and carboplatin in advanced non-small-cell lung cancer: a phase III trial-INTACT 2. J Clin Oncol 2004;22(5): 785-794

38 Gatzemeier U, Pluzanska A, Szczesna A, et al. Phase III study of erlotinib in combination with cisplatin and gemcitabine in advanced non-small-cell lung cancer: the Tarceva Lung Cancer Investigation Trial. J Clin Oncol 2007;25(12):1545-1552

39 Herbst RS, Prager D, Hermann R, et al; TRIBUTE Investigator Group. TRIBUTE: a phase III trial of erlotinib hydrochloride (OSI-774) combined with carboplatin and paclitaxel chemotherapy in advanced non-small-cell lung cancer. J Clin Oncol 2005;23(25): $5892-5899$
40 Mok TS, Wu YL, Thongprasert S, et al. Gefitinib or carboplatinpaclitaxel in pulmonary adenocarcinoma. N Engl J Med 2009;361 (10):947-957

41 Rosell R, Carcereny E, Gervais R, et al; Spanish Lung Cancer Group in collaboration with Groupe Français de Pneumo-Cancérologie and Associazione Italiana Oncologia Toracica. Erlotinib versus standard chemotherapy as first-line treatment for European patients with advanced EGFR mutation-positive non-small-cell lung cancer (EURTAC): a multicentre, open-label, randomised phase 3 trial. Lancet Oncol 2012;13(3):239-246

42 Zhou C, Wu YL, Chen G, et al. Erlotinib versus chemotherapy as first-line treatment for patients with advanced EGFR mutationpositive non-small-cell lung cancer (OPTIMAL, CTONG-0802): a multicentre, open-label, randomised, phase 3 study. Lancet Oncol 2011;12(8):735-742

43 Maemondo M, Inoue A, Kobayashi K, et al; North-East Japan Study Group. Gefitinib or chemotherapy for non-small-cell lung cancer with mutated EGFR. N Engl J Med 2010;362(25):2380-2388

44 Mitsudomi T, Morita S, Yatabe Y, et al; West Japan Oncology Group. Gefitinib versus cisplatin plus docetaxel in patients with nonsmall-cell lung cancer harbouring mutations of the epidermal growth factor receptor (WJTOG3405): an open label, randomised phase 3 trial. Lancet Oncol 2010;11(2):121-128

45 Yang JC, Schuler MH, Yamamoto N, et al. LUX-Lung3: a randomized, open label, phase III study of afatinib versus pemetrexed and cisplatin as first-line treatment for patients with advanced adenocarcinoma of the lung harboring EGFR-activating mutations. J Clin Oncol 2012;30(suppl):Abstract LBA7500

46 Kim DW, Ahn MJ, Shi Y, et al. Results of a global phase II study with crizotinib in advanced ALK-positive non-small cell lung cancer (NSCLC). J Clin Oncol 2012;30(suppl):Abstract 7533

47 Camidge DR, Bang YJ, Kwak EL, et al. Activity and safety of crizotinib in patients with ALK-positive non-small-cell lung cancer: updated results from a phase 1 study. Lancet Oncol 2012;13 (10):1011-1019

48 Tsao MS, Aviel-Ronen S, Ding K, et al. Prognostic and predictive importance of p53 and RAS for adjuvant chemotherapy in non small-cell lung cancer. J Clin Oncol 2007;25(33):5240-5247

49 Pao W, Wang TY, Riely GJ, et al. KRAS mutations and primary resistance of lung adenocarcinomas to gefitinib or erlotinib. PLoS Med 2005;2(1):e17

50 Janne PA, Shaw AT, Pereira JR, et al. Phase II double-blind, randomized study of selumetinib (SEL) plus docetaxel (DOC) versus DOC plus placebo as second-line treatment for advanced KRAS mutant non-small cell lung cancer (NSCLC). J Clin Oncol 2012;30(suppl):Abstract 7503

51 Bergethon K, Shaw AT, Ou SH, et al. ROS1 rearrangements define a unique molecular class of lung cancers. J Clin Oncol 2012;30 (8):863-870

52 Shaw AT, Camidge DR, Engelman JA, et al. Clinical activity of crizotinib in advanced non-small cell lung cancer (NSCLC) harboring ROS1 gene rearrangement. J Clin Oncol 2012;30(suppl):Abstract 7508

53 Suehara Y, Arcila ME, Dela Cruz Drillon AE, et al. KIF5B-RET: Discovery of a novel fusion oncogene in lung adenocarcinomas by a systematic screen for tyrosine kinase fusions and identification of patients for a RET targeted therapy trial. J Clin Oncol 2012;30(suppl):Abstract 7578

54 Paik PK, Arcila ME, Fara M, et al. Clinical characteristics of patients with lung adenocarcinomas harboring BRAF mutations. J Clin Oncol 2011;29(15):2046-2051

55 Marchetti A, Felicioni L, Malatesta S, et al. Clinical features and outcome of patients with non-small-cell lung cancer harboring BRAF mutations. J Clin Oncol 2011;29(26):3574-3579

56 Brahmer JR, Horn L, Antonia S, et al. Clinical activity and safety of anti-PD1 (BMS-936558, MDX-1106) in patients with advanced non-small-cell lung cancer (NSCLC). J Clin Oncol 2012;30(suppl): Abstract 7509 
198 Treatment of Late Stage Lung Cancer Scheff, Schneider

57 National Comprehensive Cancer Network ${ }^{\circledR}$, NCCN Clinical Practice Guidelines in Oncology (NCCN Guidelines ${ }^{\circledR}$ ); Non-Small Cell Lung Cancer: Version 2. 2012. Available at: http://www.tri-kobe.org/ nccn/guideline/lung/english/non_small.pdf

58 Meyerson M, Gabriel S, Getz G. Advances in understanding cancer genomes through second-generation sequencing. Nat Rev Genet 2010;11(10):685-696
59 Paik PK, Hasanovic A, Wang L, et al. Multiplex testing for driver mutations in squamous cell carcinomas of the lung. J Clin Oncol 2012;30(suppl):Abstract 7505

60 Sequist LV, Heist RS, Shaw AT, et al. Implementing multiplexed genotyping of non-small-cell lung cancers into routine clinical practice. Ann Oncol 2011;22(12):2616-2624 